\title{
A Case Series of Penile Skin Grafting in Children
}

\author{
${ }^{1}$ Department of Burns and Plastic Surgery, Children's Hospital of \\ Chongqing Medical University, Chongqing, China \\ 2 Ministry of Education Key Laboratory of Child Development and \\ Disorders, Key Laboratory of Pediatrics in Chongqing \\ (CSTC2009CA5002), Chongqing International Science and \\ Technology Cooperation Center for Child Development and \\ Disorders, Children's Hospital of Chongqing Medical University, \\ Chongqing, China \\ Eur J Pediatr Surg Rep 2020;8:e77-e80.
}

Lin Qiu ${ }^{1} \quad$ Xuan Zhang ${ }^{2} \quad$ Yan Liu $^{1} \quad$ Yuexian Fu $^{1} \quad$ Xingang Yuan ${ }^{1}$

\begin{abstract}
Address for correspondence Xingang Yuan, PhD, Department of Burns and Plastic Surgery, Children's Hospital of Chongqing Medical University, No. 136 Zhongshan Er Road, Yuzhong, Chongqing 400014, China (e-mail: 535538769@qq.com).
\end{abstract}

\begin{abstract}
Keywords

- pediatric

- penis

- skin grafts

- lymphedema

Pediatric penile skin grafting is rarely performed. We present a case series of four pediatric patients receiving skin grafting due to the loss of penile skin. The four boys were followed up for 1 to 5 years. One full-thickness skin graft and three split-thickness skin grafts (STSGs) survived well with low Vancouver scar scale scores. One boy gradually developed lymphedema of the distal foreskin and underwent a second preputioplasty. He presented with normal erectile function and did not experience any pain. We propose thick STSGs as the most appropriate choice for pediatric penile skin reconstruction. Lymphedema of the foreskin is an important long-term complication of penile skin grafting.
\end{abstract}

\section{New Insights and the Importance for the Pediatric Surgeon}

Skin grafting of the pediatric penis is not performed frequently. The aim of this study was to put forward some thoughtprovoking observations about penile skin grafting in children after considering our study results and other literature.

\section{Introduction}

Penile congenital abnormalities or traumas require adequate skin coverage for reconstruction. ${ }^{1}$ Local flaps are preferred by surgeons, but they are not always available. ${ }^{1}$ Alternatively, fullthickness skin grafts (FTSGs), split-thickness skin grafts (STSGs), and artificial dermis implants may be considered. The strategy is chosen depending on the location and size of the defect. Several reports were published on adult diseases requiring penile skin grafts. ${ }^{2}$ However, only a few reports exist on penile skin grafts in the pediatric population, as diseases that cause severe penile tissue loss appear to be rare in children. Pediatric surgeons encounter hypospadias-related complications and penile skin deficiency but often prefer to reconstruct the urethra with a tabularized (or on lay-staged) buccal mucosal graft and cover the free graft with available penile skin. ${ }^{3}$ Hence, pediatric penile skin grafting is rarely performed. We present a case series of four patients who underwent penile skin grafting at our department from 2012 to 2019.

\section{Case Report}

\section{Cases 1 and 2}

A 4.11-year-old boy and an 8-year-old boy suffered a high fall-related penile injury. The 4.11-year-old boy was treated 8 hours after injury. The defect area measured $3.0 \times 2.5 \mathrm{~cm}$. He received a FTSG after debridement because the time after received

February 5, 2020

accepted after revision

July 4,2020

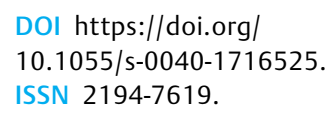

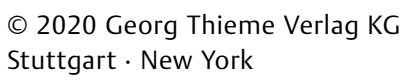

License terms

(c) (i) 


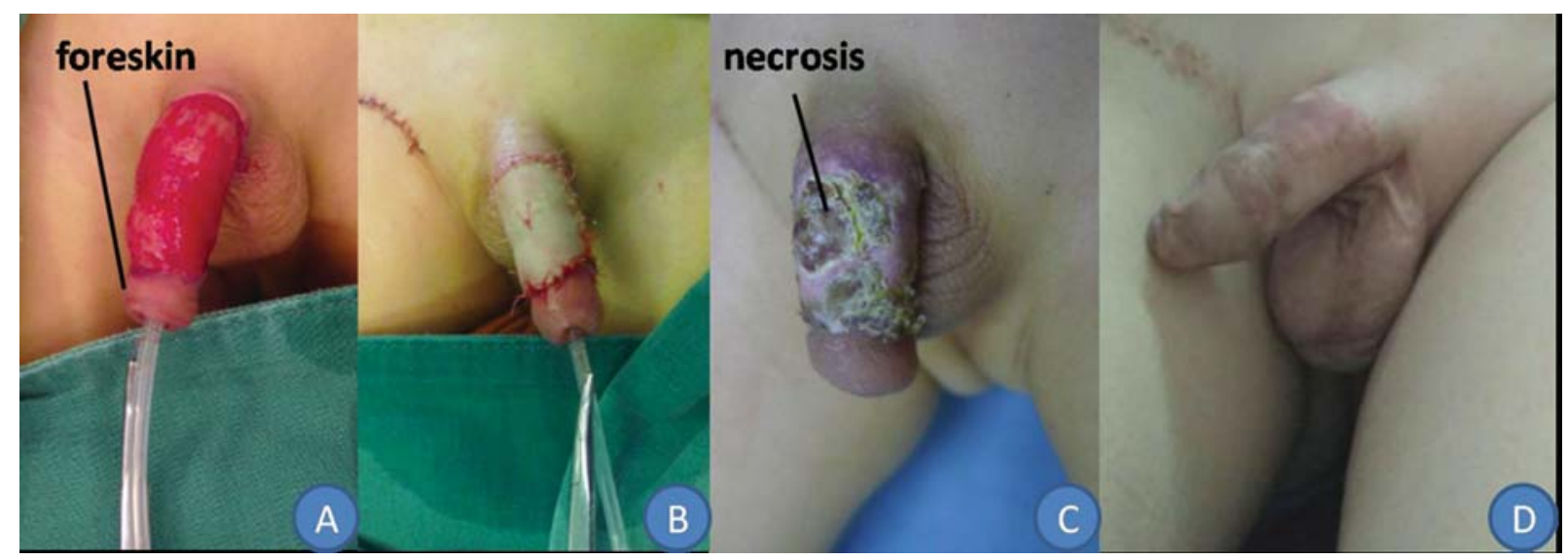

Fig. 1 A 4.11-year-old boy suffered a high fall-related penile injury and presented with tearing of the penile skin, loss of the penile shaft skin, and retention of the front of the foreskin (A). The boy received a full-thickness skin graft (FTSG) (B). The FTSG survived almost completely at 10 days after surgery, and the small area of skin necrosis healed after changing of the dressing (C). The scar was slightly hyperplastic and hyperpigmented 8 months after surgery (D).

injury was relatively short and the penile wound surface was relatively clean ( - Fig. 1B). Full-thickness skin was harvested from the lateral inguinal region. The inguinal donor wound was sutured. The dressing and the catheter were removed 10 days after the operation.

The 8-year-old boy was admitted to the hospital 24 hours after injury. The defect penile skin area measured $2.0 \times 2.8 \mathrm{~cm}$, and the defect scrotal skin area measured $3.0 \times 3.5 \mathrm{~cm}$. He received debridement and an artificial dermis implant (PELNAC, GUNZE Corporation, Japan) during the primary surgery, followed by a thick STSG 14 days after surgery ( - Fig. 2B).

\section{Case 3}

A 6.6-year-old boy, who sustained an electric cautery circumcision-related penile injury, was transferred from the urology department to the burns and plastic surgery department 10 days after injury. The defect area measured $1.5 \times 2.5 \mathrm{~cm}$. After debridement, a thick STSG was performed (-Fig. 3B).

\section{Case 4}

A 12.5-year-old boy, who suffered a traffic accident-related penile injury, received primary suturing at another hospital. The penile skin necrosis area measured $2.2 \times 3.1 \mathrm{~cm}$, and the left thigh necrosis skin area measured $38 \times 15 \mathrm{~cm}$. He was transferred to our department 10 days after injury, and he received debridement and an artificial dermis implant (acellular dermal matrix) during the primary surgery. After 14 days, the acellular dermal matrix was removed, and a 0.2-mm STSG was placed on the granulation tissue wound (-Fig. 4B).

This STSG was harvested from the scalp using a Zimmer electric dermatome. The donor site was covered with antibiotic dressing and healed naturally after 2 weeks. The penile dressing and the catheter were removed 7 days after STSG placement.

A Foley catheter was inserted and retained throughout the treatment. We used the dressing for hypospadias surgery as a reference to design the skin graft dressing. On the inner side, a mesh-like lipid hydrogel dressing (UrgoTul, Laboratories URGO, France) was evenly applied to the grafted skin so as to form a moderately tight sheath and to avoid dressing adhesion. An antibiotic ointment (mupirocin) was applied to the second layer, and the outer layer was wrapped with an elastic dressing. The graft was applied tightly to the wound surface, and the absence of dissolution or scabbing was considered to indicate good skin graft survival. The Vancouver scar scale (VSS; Baryza and Baryza, 1995) was used to evaluate the scar quality in all patients. The scale consists of the following variables: vascularity, pliability, height, and pigmentation (-Supplementary Table 1).

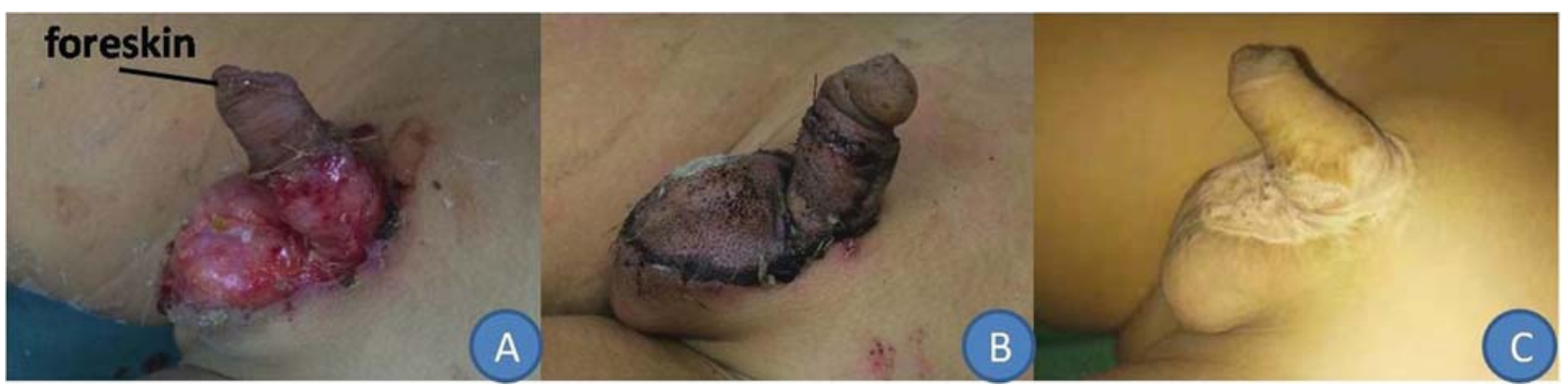

Fig. 2 An 8-year-old boy suffered a high fall-related penile injury and presented with loss of the penile shaft skin and part of the scrotal skin, and retention of the front of the foreskin (A). The split-thickness skin graft survived completely as observed 7 days after surgery (B). The scar was noted to be flat and hypopigmented 3 years after surgery (C). 


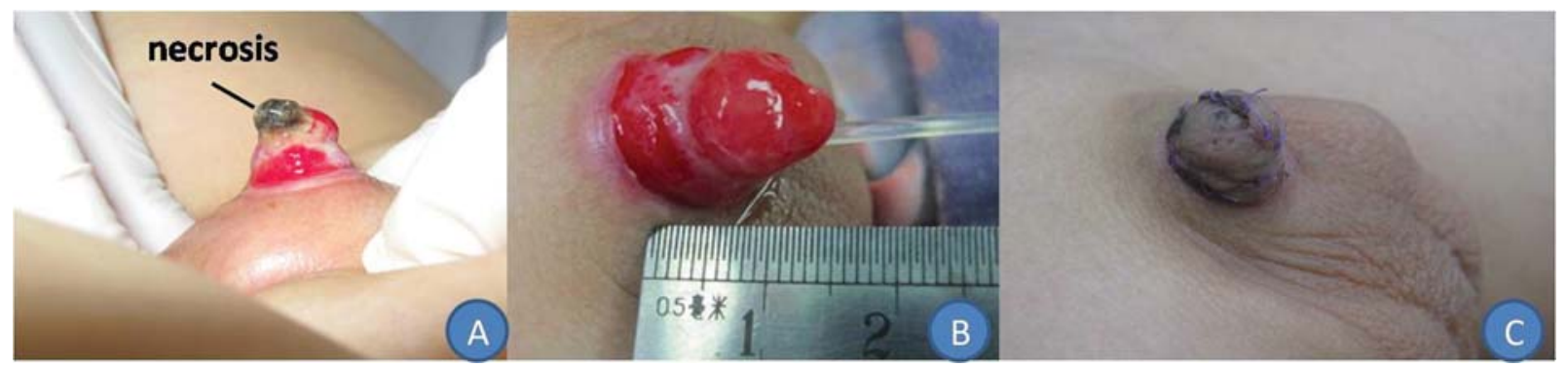

Fig. 3 A 6.6-year-old boy suffered an electric cautery ritual circumcision-related penile injury and presented with loss of the penile skin and the glans penis (A). After debridement (B), the split-thickness skin graft survived completely as observed 1 month after surgery (C).

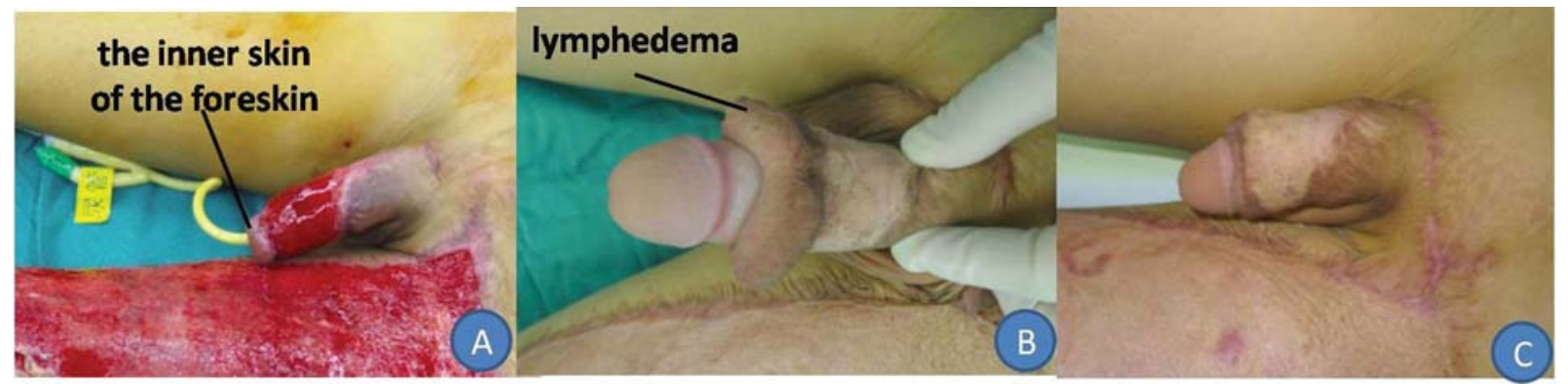

Fig. 4 A 12.5-year-old boy suffered a traffic accident-related penile injury and presented with tearing of most of the penile skin and part of the left thigh skin, and presented with retention of the inner side of the foreskin (A). The split-thickness skin graft survived completely; however, lymphedema of the distal penile skin had developed 1 year after surgery (B). After foreskin plastic surgery, the scar was observed to be flat and hypopigmented (C).

The three thick STSGs survived completely, with no signs of dissolution or scabbing (-Figs. 2, 3, and 4C). The FTSG survived almost completely, with a small area of skin necrosis healing remaining after the dressing was changed (-Fig. 1D). The 12.5-year-old boy gradually developed lymphedema of the distal foreskin (-Fig. 4B), and he received prepuce plastic surgery 6 months after skin grafting ( - Fig. 4C). He reported that he could achieve an erection and experienced no pain during urination. All patients were able to urinate easily. The VSS scores of these four patients are presented in -Supplementary Table 1. Scar vascularity and pliability in all four patients were close to those of normal skin. The scars after STSG were hypopigmented and flat, whereas the scar after the FTSG was hyperpigmented, showing slight hyperplasia $(<2 \mathrm{~mm})$. All parents were satisfied with the appearance and function of the penis.

\section{Discussion}

Some adult diseases lead to severe penis tissue loss and require skin grafting, for example, infection, trauma, burns, malignancy, skin disorders, and primary lymphedema. ${ }^{4}$ However, these conditions are rare in children. Nevertheless, we treated four children over the past 8 years whose penile skin defects were caused by trauma. In a pediatric hypospadias review, only 23 patients underwent FTSG, and 4 patients received STSG, among 215 patients; the 4 patients experienced a complete graft take, but secondary contraction and ulceration were observed and associated with sexual activi- ty. ${ }^{5}$ Thus, patients with hypospadias or "hypospadias cripples" requiring skin grafts are also relatively rare.

An ongoing debate exists about the optimal skin type to be used for penile skin grafts. A greater amount of dermis is included in FTSG than in STSG; therefore, some important distinctions in the nature and potential uses of the two types of grafts should be borne in mind. ${ }^{6}$ For FTSG exhibiting a greater metabolic demand and requiring a well-vascularized recipient site, their survival is more uncertain. In contrast, STSGs demonstrate a lower metabolic demand and require less ideal conditions for survival; therefore, STSGs exhibit a much broader application range than FTSGs. ${ }^{7-10}$ FTSGs display a higher degree of primary contraction, which is the recoil occurring immediately after the skin graft is harvested and is related to the amount of dermis elastin in the tissue. FTSGs may lose up to $40 \%$ of their surface area, whereas STSGs typically lose only up to $10 \%{ }^{11}$ Over time, FTSGs exhibit a tendency to resist secondary contraction, which refers to the contraction of a skin graft after healing. Once secondary contraction ends, FTSGs tend to stretch and grow with the individual, whereas STSGs do not tend to expand, ${ }^{11}$ which may cause contraction or scarring.

As the penile skin is thin, hairless, and flexible, and it undergoes a substantial change in size with penile erection, some researchers suggested FTSG as the preferred choice for penile skin graft. ${ }^{12,13}$ Chertin et al ${ }^{14}$ reported penile STSG in 17 children and adolescents, with 94\% graft take, with 6 sexually active patients reporting normal sexual intercourse and sensation; none of the patients experienced shrinkage of the STSG. Alwaal et $\mathrm{al}^{13}$ reported a successful outcome for 
genital STSG in 52 out of 54 adult patients, with maintained or improved erection, normal voiding, good cosmetic outcome, and normal mobility. Black et $\mathrm{al}^{9}$ reported penile STSG in nine adult patients, and all experienced a complete graft take. A satisfactory cosmetic outcome was obtained at a mean follow-up of 6 months, and erectile function and ejaculation were preserved in potent patients. In our four patients, the three STSGs showed complete graft survival, and the FTSG showed almost complete survival, with a small area of skin necrosis healing after changing of the dressing. The small area of skin necrosis may have been caused by a contaminated wound or/and be due to the change in penile size with erection, which caused poor metabolism and blood supply in the FTSG. All boys were able to urinate easily, and the 12.5-year-old boy could achieve an erection without experiencing any pain. Based on the above-listed observations and the results from our own study, thick STSG appears to be the most appropriate choice for penile skin reconstruction.

Long-term complications of penile skin grafting should be borne in mind. The 12.5-year-old boy who received an artificial dermis implant (acellular dermal matrix) and a thick STSG gradually developed lymphedema of the distal penile skin ( $\sim$ Fig. 4B) and required extra foreskin plastic surgery. Diaz et al noted ${ }^{15}$ that lymphedema may develop if any excess distal penile skin is not excised, and limiting the amount of mucosal collar or consider direct anastomosis to the glans is prudent. When loss of the proximal penile skin occurs, the tendency is to preserve any viable distal skin. However, when loss of the proximal penile skin occurs, the proximal dartos layer is usually lost, resulting in disruption of lymphatic drainage of the distal penile skin. The native skin should be resected up to the level of the glans to avoid a ring of lymphedematous skin developing around the glans. If skin loss is not circumferential, leaving the native skin in place is preferable, especially if several days of observation have not resulted in any increase in skin edema. ${ }^{6}$ The $12.5-$ year-old boy received an artificial dermis implant (acellular dermal matrix) during primary surgery, and the 8-year-old boy received an artificial dermis implant (PELNAC, GUNZE Corporation, Japan) during primary surgery. However, only the 12.5-year-old boy developed lymphedema. The PELNAC grows on the wound bed and forms granulation tissue, with a structure similar to that of the dermis. However, the acellular dermal matrix is a short-term onlay, which covers the wound bed and stimulates the growth of granulation tissue. The matrix was eventually removed. Whether the implant was the cause of the lymphedema remains unknown, and so, more cases and studies are required.

\section{Conclusion}

In conclusion, pediatric penile skin grafting is a rare procedure. Based on our experience and observations published in the scientific literature, thick STSG appears to be the most appropriate choice for pediatric penile skin reconstruction. Lymphedema of the foreskin is an important long-term complication of penile skin grafting.

\section{Conflict of Interest}

None declared.

\section{References}

1 Triana Junco P, Dore M, Nuñez Cerezo V, et al. Penile reconstruction with skin grafts and dermal matrices: indications and management. European J Pediatr Surg Rep 2017;5(01):e47-e50

2 McAninch JW. Management of genital skin loss. Urol Clin North Am 1989;16(02):387-397

3 Manzoni G, Bracka A, Palminteri E, Marrocco G. Hypospadias surgery: when, what and by whom? BJU Int 2004;94(08):1188-1195

4 Patino G, Zheng MY, Breyer BN, Cohen AJ. Skin grafting applications in urology. Rev Urol 2019;21(01):8-14

5 Fam MM, Hanna MK. Resurfacing the penis of complex hypospadias repair ('Hypospadias Cripples'). J Urol 2017;197(3 Pt 2):859-864

6 Thakar HJ, Dugi DD III. Skin grafting of the penis. Urol Clin North Am 2013;40(03):439-448

7 Asimakopoulos AD, Iorio B, Vespasiani G, Cervelli V, Spera E. Autologous split-thickness skin graft for penile coverage in the treatment of buried (trapped) penis after radical circumcision. BJU Int 2012;110(04):602-606

8 Rybak J, Larsen S, Yu M, Levine LA. Single center outcomes after reconstructive surgical correction of adult acquired buried penis: measurements of erectile function, depression, and quality of life. J Sex Med 2014;11(04):1086-1091

9 Black PC, Friedrich JB, Engrav LH, Wessells H. Meshed unexpanded split-thickness skin grafting for reconstruction of penile skin loss. J Urol 2004;172(03):976-979

10 Gillett MD, Rathbun SR, Husmann DA, Clay RP, Kramer SA. Splitthickness skin graft for the management of concealed penis. J Urol 2005;173(02):579-582

11 Corps BV. The effect of graft thickness, donor site and graft bed on graft shrinkage in the hooded rat. Br J Plast Surg 1969;22(02):125-133

12 Garaffa G, Christopher N, Ralph DJ. The management of genital lymphoedema. BJU Int 2008;102(04):480-484

13 Alwaal A, McAninch JW, Harris CR, Breyer BN. Utilities of splitthickness skin grafting for male genital reconstruction. Urology 2015;86(04):835-839

14 Chertin B, Kocherov S, Binenboym R, et al. Fenestrated sheet splitthickness skin grafting for reconstruction of penile skin loss in pediatric population. J Pediatr Surg 2016;51(08):1362-1365

15 Diaz EC, Corcoran JF, Johnson EK. Pediatric penile reconstruction using autologous split-thickness skin graft. J Pediatr Urol 2016;12 (03):185-186 